\title{
Analysis on the Shenhua Railway AxleMaintenance
}

\author{
Yang Juping \\ Shenhua Railway Freight Transport Co., Ltd.,China
}

Keywords: freight train, wheel, bearing, life, material, maintenance and application, monitoring.

\begin{abstract}
The main problems in the maintenance of Shenhua Railway axles are the short service life of the wheels, the early failure of the bearings, the standard of the ultrasonic inspection of the axles is not uniform, and the $5 \mathrm{~T}$ networking is not satisfactory. It is necessary to carry out comprehensive test research on wheel life, so as to improve the reliability of bearing operation, strengthen the guidance on the inspection of axles, and accelerate the integrated networking process of 5 T equipment.
\end{abstract}

\section{Introduction}

The axle is a key component of the railway vehicle's moving parts. Its maintenance quality and management level causes a direct impact on driving safety. Shenhua Railway Freight TransportCo., Ltd. is currently the largest railway self-provided freight transport specialization company in China. By the end of 2017, it managed 49,855 self-owned freight trains. The main models are C64, C70, C80 and KM98 type open-top trains. The main axle models for maintenance are RD2, RE2B and RF2 domestic main axles. There are four maintenance branches engaged in the first, second and third stage of the axle, one branch engaged in therepairing of third and fourth stage of the axle and the general maintenance of the bearing.Each applicationline is rationally arranged, and the relevant $5 \mathrm{~T}$ equipment is used for online monitoring of the axles, which forms a comprehensive maintenance system that includes the axle inspection, repairing, processing, assembly and bearing maintenance to ensure the safe operation of Shenhua Railway Freight transportations.

However, due to various factors such as the complicated Shenhua railway lines, high operating density, and the operation of 10,000-ton trains, a series of problems have been exposed during the inspection and running of the axles, which must be highly valued.

\section{Existing problems}

\subsection{Wheel life is short}

\subsubsection{The thickness of wheel rim prototype is not qualified}

After the railway wagon wheel was upgraded by $120 \mathrm{~km} / \mathrm{h}$, the prototype of the wheel rim thickness was changed from the original $65 \mathrm{~mm}$ to the current $50 \mathrm{~mm}$, which reduced the weight of the wheel and optimized the dynamic performance.It is called speed increasing and weight reduction wheelset, mainly to meet the needs of freight train speed-up to $120 \mathrm{~km} / \mathrm{h}$.However, the characteristics and transportation mode of Shenhua Railway Line shows that the freight train operation is more suitable for the 10,000 tons of heavy haul trains. The train running speed is controlled under $80 \mathrm{~km} / \mathrm{h}$, the wheel rim thickness section repairing limit is $26 \mathrm{~mm}$, and the abrasion and processing allowance is only $24 \mathrm{~mm}$. Due to the complicated running conditions of the wheels, a large part of the wheelsets need to be repaired by the vehicle factory and the section repairing, and the running wear and repairingis restored to the tread shape, which causes the thickness of the rim to be reduced faster.According to the statistics madeby the maintenance site, most of the wheels have reached the limit of rim scrapping after running for 8-12 years, and they are about $6 \%$ of the total number of inspection wheels, which greatly wastes maintenance costs. However, the wheel with a rim thickness prototype of $65 \mathrm{~mm}$ can be used for more than 18 years. 


\subsubsection{Wheel material needs further improvement}

The speed increasing and reconstruction of the wheel is for the development of railway freight transports. The heavy load of the vehicle increases the axle load, causing the corresponding wheelrail contact stress increases, the contact area increases, and the contact fatigue failure and the abrasive wear also increase.As the size and weight of the wheels change, it is necessary to update the materials immediately.

At present, most of the materials used in railway freight train wheels are CL60 type wrought steel wheels and ZL-B type cast steel wheels. The wheels of these two steel types are still incomplete in terms of wheel strength, wear resistance and thermal crack resistance, and fail to achieve uniform abrasion of the wheel tread.The wheels must be further improved in matching with rail hardness.

\subsubsection{The abrasion of the tread parts is significant during the running of the wheels}

According to statistics, at present, the wheels of Shenhua Railway freight trains enter the peak period of wheel tread failures in about 1.5 years, and a large number of wheels have worn more than the limit.In 2017, Shenhua Company experienced a total of 2,415 faults in the circumferential wear of the treads. However, in 2018, there were 2,214 faults occurred from January to June, showing a clear upward trend. This is a bottleneck problem for the development of train types and status repairing with long inspection cycles.Take the RE2B type axle mounted on C80 freight train as an example, during the sectionrepair period, the rotation repairingfor the wheel pairs is about $80 \%$.The main reasons areexcessive wearing of the circumference, peeling of the tread, partial depression of the tread and exceeding of the wheel diameter difference.The details for main reasons are as follows: the length of the 20,000-ton train was lengthened, the braking distance and time were extended during train braking, the adhesive friction time of the brake shoe and the wheel was prolonged, the friction force was increased, and during the braking period, the wearing of the surface of the brake shoe and the circumference of the wheel tread would also be relatively increased, which was bound to increase the wearing of the circumference of the wheel tread.Some vehicles have poor braking, the brake shoes and the vehicle tread badlywore for a long time, the wheel pairs alwaysslid along the rail for a long distance, and the sliding friction caused the wheel tread to wear and partially dent. When the train runs on a huge downhill road, most of the heat load generated by the vehicle during frequent braking is absorbed by the wheel tread, and the circumference of the wheel tread is repeatedly subjected to the physical process of heating-cooling-reheating-re-cooling.As a result, the circumference of the wheel tread is prone to fatigue cracks and forms layer-by-layer peeling, which eventually leads to circumferential wearing and peeling of the tread surface.Due to the small number of turning stations on the dedicated line, the vehicles have no chanceto turn around in a short time, causing the wheel diameter difference of the left and right wheels of a large number of wheel sets to exceed the limit.

\subsubsection{Internal defects of the wheel}

During the appearance inspection on the inspection site and the wheel rotaterepairing, a large number of wheels were found to have internal defects.Since January 2018, Shenhua has found that there are 68 internal defects during the wheel repairing, mainly for wheel rim depth cracks and wheel tread hot cracks.These cracks are difficult to detect byappearance inspection, but during therotate repairing, as the cutting depth increases, the cracks will gradually be exposed. Those cracks will be found more and more in the deep, some directly reach the rim limit, and a large area of interlayer defects has also appeared inside individual wheels. The main reason is that arounda certain depth range under the wheel contact surface (generally 15 to $20 \mathrm{~mm}$ below the wheel-rail contact surface),there are some large-sized chain inclusions.Due to the difference between the elastic properties and the thermal properties between the inclusions and the base metal of the wheel, a non-uniform stress field is generated under the contact stress of the wheel and rail and during the cooling process, which is subjected to a larger load than the surrounding base material, causing the stress in the vicinity of the inclusions to be lowered.Meanwhile, the thermal barrier coefficient of 
the inclusions is different from the surrounding base metal, also resulting in different speeds of shrinkage, and tensile stress is generated at the boundary between the base metal and the inclusions.Under the action of wheel-rail contact stress, first, stress concentration occurs at the pole of the inclusions, causing the inclusions to be separated from the base material or breaking the tightly-bonded inclusionsto form cavitation.Then, in the process of continuing to load, the concentration of stress is greater and cracks are generated here, and the faster the train runs, the faster the cracks propagate.The tread brake hot cracksare caused by thermal damage caused by brake shoe contact during braking. The bad frictional contact conditions of brake shoes and the bad function of brake system will cause the wheel tread to be bear high thermal load partially. With the increasing and decreasing of temperature cycle, the high temperature resistance will be reduced to form thermal fatigue. It eventually causes cracks in the heated area of the tread.If these two types of cracks are found to be untimely, the malignant driving accidents such as collapse and derailment may occur, which cause the significant effectto the running safety of trains.

\subsection{Bearings fail significantly in the early stages}

\subsubsection{Bearing peeling failure}

Rolling bearings for railway wagons often have early failures (or abnormal failures) due to internal or external factors that do not reach their calculated life under certain conditions of use.Statistical analysis shows that under normal conditions, the failure modes of rolling bearings are mostly fatigue failure, accounting for more than $70 \%$ of the total failure. Shenhua railway bearings are used in harsh conditions and bearing damage occurs frequently. The damages of railway rolling bearings in China can be divided into 7 types, they have various forms, and the reasons are complicated, but the peeling damages are the most of all.Peeling is the phenomenon that the surface of the part is peeled off by metal sheet or block under the action of high contact stress cycle.The parts resulting from the peeling are the bearing inner and outer ring raceway surfaces and the roller rolling surfaces.In the actual work of the rolling bearing, the rolling element and inner ring(or outer ring) bears the alternating load, and the contact stress is large due to the small contact area.Under the repeated action of high rotational speed and large alternating contact stress, the metal will experience contact fatigue and the surface will be partially peeled off.Contact fatigue is the same as general fatigue, and they will alsoexperience two stages: crack formation and expansion.Crack formation is the result of repeated partial plastic deformation of the metal, depending on the comparative relationship between the maximum comprehensive shear stress in rolling contact parts and the yield strength of materials.According to the comparison of the distribution of maximum comprehensive shear stress and the distribution of material strength, the location of crack generation and the types of contact fatigue can be determined. The types of contact fatigue can be divided into three types: hard spotspeeling (pitting), shallow peeling and deep peeling (surface crushing). These types of peeling often occur during the repairing period, and most of them are found in the thirdlevel repairing of the axle, andthe running time or mileage has not reached the design data. It is difficult to find such faults in various monitoring equipment along the running lines. Therefore, after the faults accumulate to a certain extent, it is easy to cause more serious traffic safety accidents.

\subsubsection{Bearing service life needs to be further improved}

The four maintenance branches of ShenhuaFreight Transport Company reached 18080 and 17014 sets of new and overhauled bearings for 352226X2-2RZ in 2017, and 34131 and 21204 sets of new and overhauled bearings for 353130B.Except for bearings that have alreadyreached the service life and cannot be repaired(such as scratches and peeling), the 352226X2-2RZ new and overhauled bearings have a total of 15,142 sets, and the 353130B new and overhauled bearings have a total of 18,339 sets, which have not reached the service life due to early failure (or abnormal failure). Their unloading caused a huge cost loss.

\subsubsection{Running mileage cannot be measured}

"Rules for the Assembly and Maintenance of Railway Freight TrainAxles" stipulates that when 
the bearings reach the specified time and mileage, they shall be repaired or scrapped accordingly.However, due to the fact that the running mileage of the bearing cannot be effectively measured according to the current means, the implementation of this regulation is not satisfactory.Since most of the freight trains in the Shenhua Railway system operate frequently, the calculation is executed according to the running mileage in the early stage. The detailed method is to calculateas 200,000 kilometers per year, and figure out the actual mileage used for bearing life judgment.However, as the frequency of transportation was affected by fluctuations in the coal market, the relevant regulations ofmaintenance based on mileage were cancelled in the later period, and the itwas replaced by the maintenance based on years. Now, the coal market has recovered and the operating frequency has increased significantly.Frequent changes are more likely to cause disturbances in the measurement of bearing life, resulting in overdue service or cost waste.

\subsubsection{Bearing accessory failure}

During the bearing overhaul period, it is often found that there are a large number of faults in the bearing accessories that affect the safety of driving. There are mainly problems such as looseness of the bearing seal cover or oil seal, loose rear bumper, lack of grease, and damage to the front cover.According to statistics, such faults account for about $30 \%$ of bearing repair failures, which easily lead to deterioration of the hot shaft and bearing running quality. In severe cases, driving accidents may occur.The main reason is that the process execution is not thorough and the quality control is not strict in bearing manufacturing. For example, the magnitude of interference of oil seal and the seal cover are insufficiently, and the grease filling does not meet the requirements; there are also some mechanical damages during operation, such as the phenomena of bumping the front cover and oil seal of bearing; the implementation of process standards for plant and section repairingis not satisfactory,for example, the rear bumper is pressed randomly without selection, which causes loosening and then wear with bearing or dust-proof plate seat.

\subsection{The standard of axle ultrasonic testing is not uniform}

After the implementation of the new "Regulations for Inspection and Repairing of Railway Wheel Axle", some new standards for ultrasonic testing of various parts of the axle are proposed, including new test block usage methods, new probe angles, sensitivity determination methods and quality judgment standards, the new B/C type that can show the automatic ultrasonic flaw detection technology of the axle, which clarifies the definition of the large axial crack of the axle, etc., giving the maintenance unit a large autonomous space.However, due to the different flaw detection equipment,and the technical standards are not uniform, and the working quality of the flaw detectors is not uniform, the branches have different understandings of the flaw detection standards, and they must be standardized and unified.

\subsection{T networking is not in place}

The 5t equipment along the Shenhua Railway lines plays a key role in the operation safety of Shenhua Railway. A large number of driving equipment faults are discovered and processed in time to make up for the shortcomings of manual operations.However, the existing 5T system still has some shortcomings in the integrated networking, mainly because the fault data cannot be coordinated with the maintenance system and prompt each other.The ideal mode is that before the vehicle factory and repairing section repairs, the maintenance personnel can see on the maintenance system which major faults have been predicted and processed during the operation of the vehicle, and confirm on-site whether the treatment is in place, whether it is necessary to do the majorrepairing in the factory and therepairing section. The main faults handled by the plant andrepairing section are related to the freight train security management system and the freight train information management system, so that the operation department along the line can see the main fault information from the maintenance, so as to prevent the faults. 


\section{Methods and recommendations}

\subsection{Conducting a comprehensive test of wheel life}

Based on a large number of on-site investigations, we can set up a special project to systematically study the material, appearance size and maintenance cycle of the wheels to find the best solution.For example, the current wheel material experiment on the Daqin line, the newly developed CL70 material of the rolling steel wheel is reduced by 30\% compared with the CL60 material of the rolling steel wheel, and the wheel wear area is reduced by $32 \%$. It is recommended that Shenhua Railway promote the use of them as soon as possible.Meanwhile, it is necessary to combine the characteristics of Shenhua Railway with the relevant wheel design department and production department to develop the wheels suitable for the operation of Shenhua line heavy-load and general-speed freight trains.

Based on the research of vehicle condition repairing, we shallreasonably determine themaintenance content, time and node of the vehicle maintenance and repair according to the characteristics of the wheel wearing, and handle the wheel failures by maintenance operation timely and effectively.

\subsection{Improve bearing operation reliability}

\subsubsection{Develop a scientific and reasonable bearing maintenance system}

According to the actual situation of the Shenhua Railway line vehicle operation, we shall formulate a scientific method for measuring the bearing mileage, accurately calculate the mileage of the bearing, and guide the appearance inspection in the bearing repair time of the vehicle factory to ensure the reliable operation of the bearing.

\subsubsection{Carry out the bearing material research}

We shall collect defective bearings, carry out special laboratory analysis, and draw more scientific and accurate conclusion, and feedback relevant research results to manufacturers and research institutes to further improve the manufacturing quality of bearings.We can effectively solve the problem of early failure due to defects in the bearing material and caused by manufacturing process.

\subsubsection{Strengthening maintenance quality and operation monitoring}

By configuring advanced equipment, we can strengthen the quality of axle maintenance and operation monitoring management, so as to improve the ability of timely detection and treatment of axle faults and ensure the efficient and safe operation of vehicles.

We can use the acoustic sensor array and adaptive technology, fault diagnosis and intelligent identification technology in TADS system to develop bearing fault diagnosis equipment with advanced technology, reasonable economy and suitable for repairing operation area.We shalluse equipment for automatic detection to further enhance the accuracy of fault diagnosis.Meanwhile, it is necessary to strengthen the acoustic diagnosis and axle temperature detection of rolling bearing fault in operation to enhance the stability, reliability and safety of the system.

\subsubsection{Improving service life of bearings}

We shall implement the requirement of "Rule 4.1.7 on Installation, Maintenance and Management of Railway Freight Wheel Bearing: If all types of newly-built bearings, overhaul and overhaul bearings fail to meet the prescribed service time or operation mileage, they must undergo general maintenance".The general maintenance of the bearing can improve the bearing repair rate and greatly improve the service life of the bearing. Therefore, the general maintenance work of the bearing should be vigorously promoted. 


\subsection{Strengthen the guidance on the flaw detection of axles}

\subsubsection{Make discussions on the process of axle flaw detection and unify the technical standards}

We shall conduct extensive research on the new flaw detection process methods stipulated in the new "Rules for the Repairing and Maintenance of Railway Freight Train Wheels and Axles", fully listen to the opinions of the frontline detectors, and hold special seminars to unify the detection proposals.For example, we add a scan range verification defect to the root of the test piece (unloading groove) based on the sensitivity verification defect, and it can not only verify the sensitivity, but also verify the scanning range of the small-angle probe. Meanwhile, we can verify whether the scanning method of the small-angle manual inspection is correct.

\subsubsection{Promote the reliable and technologically advanced flaw detection equipment}

The advanced ultrasonic phased array technology, compared with traditional ultrasonic testing technology, has the following advantages:

1) It uses a single-axis sector scan instead of a grid scan, which can improve the detection speed.

2) It is possible to scan thick and large parts and various shapes of complex parts without moving the probe or moving the probe as little as possible, which is an effective means to solve the problem of poor accessibility and space limitation.

3) Generally, multi-angle and multi-directional scanning of the entire volume or area of interest can be realized without complicated scanning device and without changing the probe.

4) It optimizes the control of the focal length, focus size and sound beam direction, and has certain advantages in terms of resolution, signal-to-noise ratio, and defect detection rate. At present, the technology has achieved good results in trial operation by some maintenance units, and it should be promoted as soon as possible.

\subsubsection{Accumulate the fault detection wheelsets and organize the discussion on theexperience of discovering the crack}

Axle flaw detection is a job that requires a high level of work experience.In the process of maintenance, there are often a large number of faulty axles with unknown conditions. In some maintenance units, the authenticity of the judgment results cannot be known in time.We should establish a fault feedback and coordination system, accumulate the fault wheel pairs, verify the flaw detection results in time for the fourth stage repairing of the axles, and regularly report the judgment results. We should often hold the technical exchange meetings to discuss the faulty axles found by the flaw detection and improve maintenance experience.

\subsection{Accelerate the integrated networking process of $5 \mathrm{~T}$ devices}

We should cooperate with relevant manufacturers as soon as possible to speed up the interconnection of $5 \mathrm{~T}$ equipment and ensure that information can be shared in time and before the axle repairing, the workers can easily find out which faults have been discovered and handled in the information system, and whether there are tread defects, whether there happened to be forecast information such as the overheating axis to determine the major repairing work. In this way, multiple information systems can be independent of each other and related to each other, and strive to achieve the main fault data of the assembly, operation and maintenance of the axle at one time when the departments query, and track the faulty axle at any time.Based on it, we can determine the repair time, processing methods, and lay the foundation for the smooth development of state repair work.

\section{Conclusion and suggestion}

(1)We shall conduct a comprehensive test of wheel life.It is recommended that Shenhua Railway promote the use of CL70-type steel-rolled wheels as soon as possible, and develop wheels suitable for the heavy-load and general-speed of Shenhua Railway. Meanwhile, we should study to improve the bearing material. 
(2) We shall formulate scientific bearing mileage measurement methods, accurately calculate bearing mileage, configure advanced equipment, strengthen axle maintenance quality and operation monitoring management, and improve the ability to detect and handle axle failures in time to ensure efficient and safe operation of vehicles.

(3) We shall vigorously promote the general maintenance work of bearings, improve the bearing repair rate and the service life of bearings.

(4)We shall conduct discussion onthe axle inspection process and unify the technical standards.

(5)We shall speed up the comprehensive networking process of 5T equipment, ensure the timely sharing of information between maintenance department and application department, so as to lay the foundation for the smooth development of repairing work.

The quality and management level of axle maintenance has a direct impact on driving safety. A complete system of axle inspection, repair, machining, assembly, bearing overhaul and axle application monitoring must be formed by us to ensure the safe operation of Shenhua Railway Freight Trains.

\section{References}

[1] Nowlan, F.S. and Heap , H. F. . Reliability - centered Maintenance[ Z] . AD/ A066 579. 1978

[2] Blake, D. C. , New Strategy for Rolling Stock Maintenance andManufacture. Rail Inten. 1990 (3) : 12 20

[3] Pergande , H , G. , Tendenzen der ModernenSienenfahrzeug-instandhaltung. ETR. 1996 (4): 171 $\sim 176$

[4] Martinse , W. O. ; Rahn, T. . ICE High - techonrails[ M] . Hes-tra- Verlag, 1991

[5] Laurent Troger.Next Generation Maintenance \& Maximo in Transit Rail [R] .France, 2011.

[6] UIC High Speed..Necessities for future high speedrolling stock[R]. 2010 